\title{
Non-obstetric complications in preeclampsia
}

\author{
Katarzyna Pankiewicz ${ }^{1}$, Ewa Szczerba ${ }^{2}$, Tomasz Maciejewski ${ }^{1}$, Anna Fijałkowska ${ }^{2}$
}

${ }^{1}$ Department of Obstetrics and Gynaecology, Institute of Mother and Child, Warsaw, Poland

${ }^{2}$ Department of Cardiology, Institute of Mother and Child, Warsaw, Poland

\begin{abstract}
Preeclampsia is a multisystem disorder of pregnancy that remains a leading cause of maternal and foetal morbidity and mortality. It is still an underestimated risk factor for future cardiovascular, cerebrovascular, and kidney disease, developing often in the perimenopausal period of a woman's life. It remains unclear whether preeclampsia is an individual risk factor for future cardiovascular, cerebrovascular, and renal events or an early marker of women with high-risk profiles for these diseases. Risk factors for cardiovascular disorders and preeclampsia are very similar and include the following: obesity, dyslipidaemia, insulin resistance, pro-inflammatory and hypercoagulable state, and endothelial dysfunction. Thus, the pregnancy can only be a trigger for cardiovascular alterations that manifest in development of preeclampsia. On the other hand, there is strong evidence that changes in cardiovascular, endothelial, and metabolic systems occurring in the course of preeclampsia may not fully recover after delivery and can be a cause of future disease, especially in the presence of other metabolic risk factors regarding, for example, perimenopause. In this review the authors present current knowledge about short- and long-term maternal consequences of preeclampsia, such as: cardiovascular disease, cerebrovascular incidents (posterior reversible encephalopathy and stroke), kidney injury (including the risk of end-stage renal disease), liver failure, and coagulopathy (thrombocytopenia and disseminated intravascular coagulation).
\end{abstract}

Key words: preeclampsia, cardiovascular disease, stroke, kidney disease, coagulopathy.

\section{Introduction}

Preeclampsia is considered to affect about $5-8 \%$ of all pregnancies. It is defined in accordance with the European Society of Cardiology (ESC) guidelines on the management of cardiovascular diseases during pregnancy 2018 as hypertension (blood pressure $\geq 140 / 90 \mathrm{~mm} \mathrm{Hg}$ ) developing after 20 weeks' gestation accompanied by a new-onset of significant proteinuria (>0.3 g/24 h) [1]. In other definitions - American College of Gynaecologists and Obstetricians (ACOG) a new-onset proteinuria is not necessary to diagnose preeclampsia instead, hypertension can be accompanied by one of the following: signs of liver or renal dysfunction, cerebrovascular or cardiovascular incidents, and foetal growth restriction (FGR). Early-onset preeclampsia is defined as disease developing before 34 weeks' gestation, whereas late-onset preeclampsia is defined as disease developing at or after 34 weeks' gestation [2].

\section{Pathophysiology of preeclampsia}

The underlying mechanism for preeclampsia is thought to be impaired placentation due to inadequate trophoblastic invasion of the maternal spiral arteries. On the basis of recent studies, preeclampsia is considered as a two-stage disorder. The first stage is reduced placental perfusion due to failed remodelling of the maternal vessels. It results in the imbalance between proangiogenic and antiangiogenic factors, a consequence of which is endothelial damage leading to the second stage: development of acute maternal syndrome with systemic multiorgan dysfunction [3].

Apart from antiangiogenic state, which seems to be one of the most important factors, there are a lot of other pathogenetic mechanisms implicated in preeclampsia, including: oxidative stress, the presence of type- 1 angiotensin II receptor $\left(\mathrm{AT}_{1}\right)$ autoantibodies, platelet and thrombin activation, and intravascular inflammation [3].

An antiangiogenic state in preeclampsia manifests as an overexpression of soluble fms-like tyrosine kinase 1 (sFlt-1), which is a soluble form of type-1 receptor for vascular endothelial growth factor (VEGF). sFlt-1 binds VEGF and placental growth factor (PIGF) and causes defective angiogenesis and endothelial dysfunction. VEGF and PIGF are very important for the maintenance of endothelial cell function, especially in fenestrated endothelium, which is found in the brain, liver, and glomer- 
uli. In preeclampsia maternal plasma concentrations of sFlt-1 are significantly increased, even prior to clinical diagnosis of disease, whereas levels of VEGF and PIGF are significantly decreased [3].

A second antiangiogenic factor implicated in the pathogenesis of preeclampsia is soluble endoglin (sEng), a cell-surface co-receptor of TGF- $\beta$ (tumour growth factor $\beta$ ) that induces migration and proliferation of endothelial cells. Maternal plasma concentrations of sEng in preeclampsia are also significantly higher than in healthy controls. Oxidative stress and anti-AT ${ }_{1}$ autoantibodies can stimulate placental production of antiangiogenic factors and additionally contribute to impaired placentation and development of preeclampsia [3].

The pathophysiology of preeclampsia is summarised in Figure 1.

\section{Screening of preeclampsia}

Knowledge about the mechanisms responsible for the development of disease led to the introduction into clinical practice screening methods of preeclampsia. This screening is performed in the first trimester, during routine 11-13-week ultrasound examination, and includes Doppler evaluation of blood flow in uterine arteries and measurement of maternal plasma concentrations of several factors, such as PIGF, sFlt-1, sEng, and PAPP-A.

The combination of maternal risk factors (history of preeclampsia, multiple pregnancy, obesity, black race), ultra-

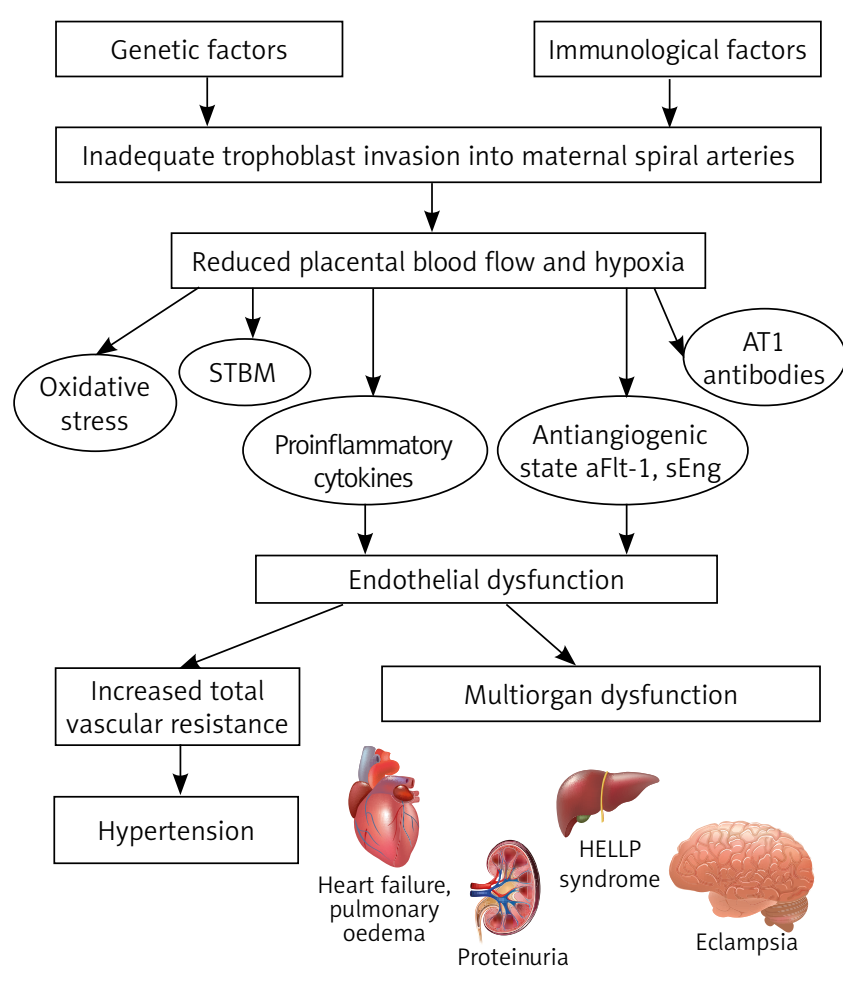

Fig. 1. Pathophysiology of preeclampsia sound indices, and biochemical measurements can reach a positive predictive value for developing preeclampsia later in pregnancy even at the level of about $96 \%$. Patients with high risk of preeclampsia can receive acetylsalicylic acid prophylaxis, which can reduce that risk. This is, however, not clinically relevant to low-risk women [4].

\section{Epidemiology and complications in preeclampsia}

In developed countries preeclampsia is a leading cause of maternal and foetal morbidity and mortality. It is responsible for about $16-18 \%$ of maternal perinatal deaths and up to $40 \%$ of foetal and neonatal deaths.

The most common complications occurring in the course of preeclampsia are: preterm delivery, foetal growth restriction (FGR), intrauterine foetal death (IUFD), and HELLP syndrome with high risk of liver rupture and eclampsia.

A very serious problem is the onset of severe maternal multiorgan dysfunction, including: heart failure, peripartum cardiomyopathy, pulmonary oedema, disseminated intravascular coagulation (DIC), cerebrovascular incidents (posterior reversible encephalopathy syndrome and stroke), acute renal failure, and liver failure.

All these complications (see Table 1) can cast a long shadow on maternal health in the future, years after delivery $[1,2]$.

\section{Cardiovascular short- and long-term complications in preeclampsia}

The most common cardiovascular complications associated with preeclampsia are: heart failure, peripartum cardiomyopathy, pulmonary oedema, and increased cardiovascular risk in future life [5].

\section{Heart adaptation during pregnancy}

During normal pregnancy, the cardiovascular system undergoes a lot of haemodynamic changes in order to support the developing foetal-placental unit.

Total peripheral resistance decreases and consequently blood pressure falls, with the greatest decrease in the second trimester (between 16 and 20 weeks). Cardiac output increases by $30-50 \%$ during the first two trimesters and plateaus after 20 weeks of gestation. It is achieved by an increase in heart rate (10 bpm on average) and a 50\% rise in plasma volume. These changes are accompanied by a transient left ventricle (LV) eccentric hypertrophy as a compensatory mechanism of volume overload. All these changes fully recover postpartum. However, pregnant women can complain about easy fatigue, dyspnoea, exercise intolerance, and oedema. These symptoms are quite common during pregnancy, but they may mask the presence of underlying LV dysfunction [6]. 


\section{Hypertension - current treatment options}

Hypertension is an essential part of preeclampsia and contributes to development of other complications, such as heart failure, renal dysfunction, or cerebral injury.

Drug treatment of severe hypertension in pregnancy is required and beneficial, whereas treatment of less severe hypertension is controversial. Although it might be beneficial for mothers with hypertension to reduce their blood pressure (BP), a lower BP may impair uteroplacental perfusion and foetal growth. The benefits and risks of antihypertensive therapy for mild to moderate hypertension (defined as systolic blood pressure SBP of 140-169 mm $\mathrm{Hg}$ and diastolic blood pressure [DBP] of 90-109 $\mathrm{mm} \mathrm{Hg}$ ) are still discussed.

Current ESH/ESC guidelines recommend initiation of drug treatment at BP of $140 / 90 \mathrm{~mm} \mathrm{Hg}$ in women with gestational hypertension, pre-existing hypertension with superimposed preeclampsia, or hypertension with subclinical organ damage or symptoms at any time during pregnancy. In any other circumstances drug treatment is recommended if SBP $\geq 150 \mathrm{~mm} \mathrm{Hg}$ or DBP $\geq 95 \mathrm{~mm} \mathrm{Hg}$ [1].

In contrast, ACOG guidelines recommend, for women with mild gestational hypertension or preeclampsia with a persistent BP of less than $160 \mathrm{~mm} \mathrm{Hg}$ systolic or $110 \mathrm{~mm} \mathrm{Hg}$ diastolic, that antihypertensive drugs should not be administered [2].

Acute-onset, severe systolic $(\geq 160 \mathrm{~mm} \mathrm{Hg}$ ) or diastolic ( $\geq 110 \mathrm{mg}$ ) hypertension that is persistent for 15 minutes or more is considered as an emergency and demands immediate treatment, because it can cause central nervous system injury. It is very important to remember that the goal is not to normalise $\mathrm{BP}$, but to achieve a range 140-150/90-100 mm Hg in order to prevent repeated, prolonged exposure to severe systolic hypertension, with subsequent loss of cerebral vasculature autoregulation and at the same time not to cause obstetric complications related to decreased uteroplacental blood flow, such as placental abruption or immediate foetal deterioration leading to IUFD [1, 2].

\section{Heart failure during preeclampsia and the postpartum period}

In women who develop preeclampsia, inadequate trophoblast invasion into maternal spiral arteries leads to impaired transformation of maternal vessels and uteroplacental circulation remains high-resistant with lower blood flow. An antiangiogenic state cause general vasoconstriction. In contrast to normal pregnancy, total vascular resistance and vascular stiffness in patients with preeclampsia are increased and cause a pressure overload of the maternal heart, which leads to LV remodelling and diastolic dysfunction [7].
Table 1. Most common obstetric and non-obstetric complications in preeclampsia.

\begin{tabular}{|c|c|}
\hline $\begin{array}{l}\text { Obstetric complications } \\
\text { in preeclampsia }\end{array}$ & $\begin{array}{l}\text { Non-obstetric complications } \\
\text { in preeclampsia }\end{array}$ \\
\hline $\begin{array}{l}\text { Intrauterine growth restriction } \\
\text { (IUGR) }\end{array}$ & Heart failure \\
\hline Intrauterine foetal death (IUFD) & Peripartum cardiomyopathy \\
\hline Preterm delivery & Pulmonary oedema \\
\hline HELLP syndrome & $\begin{array}{l}\text { Future risk of cardiovascular } \\
\text { disease }\end{array}$ \\
\hline \multirow[t]{8}{*}{ Eclampsia } & $\begin{array}{l}\text { Posterior reversible } \\
\text { encephalopathy syndrome }\end{array}$ \\
\hline & Stroke \\
\hline & Renal failure \\
\hline & Acute kidney injury \\
\hline & $\begin{array}{l}\text { Future risk of end-stage renal } \\
\text { disease }\end{array}$ \\
\hline & Liver failure \\
\hline & Hepatic rupture \\
\hline & $\begin{array}{l}\text { Coagulopathy (i.a. } \\
\text { thrombocytopaenia and DIC) }\end{array}$ \\
\hline
\end{tabular}

A typical finding in women with preeclampsia is mild-to-moderate isolated LV diastolic dysfunction accompanied by LV concentric hypertrophy. LV remodelling is asymmetrical, involving predominantly the basal anteroseptum and is a response to the increased systemic afterload in order to minimise myocardial oxygen demand and preserve LV function [6].

Diastolic function abnormalities may also play a significant role in the pathogenesis of pulmonary oedema. It is a life-threatening condition, developing mainly in the early post-partum period in the setting of haemodynamic changes and fluid resorption. In a recently published study pulmonary oedema occurred in $9.5 \%$ of patients with severe preeclampsia [5].

Global systolic function is preserved in the majority of women with preeclampsia. However, acute heart failure with reduced ejection fraction may also occur, especially as a result of hypovolaemia from haemorrhage, intracerebral haemorrhage, or the adverse effect of pharmacological agents. A rare cause of acute heart failure in preeclampsia is myocardial infarction related to spontaneous coronary artery dissection [5-7].

Several studies assessing the relationship between signs of impaired placentation (e.g. altered uterine arteries Doppler indices), cardiovascular profile, and development of preeclampsia revealed that women with both placental insufficiency and LV dysfunction were more likely to develop preeclampsia than those with placental insufficiency but normal LV function. It shows that the ability of the maternal cardiovascular system to adapt to placental dysfunction plays an important role in the development of preeclampsia [7]. 


\section{Peripartum cardiomyopathy}

Peripartum cardiomyopathy (PPCM) is defined by the European Society of Cardiology as cardiomyopathy with reduced ejection fraction $(<45 \%)$ presenting towards the end of pregnancy or in the first four months postpartum in a woman without previously known structural heart disease [8].

A recent meta-analysis of 22 studies with almost 1000 cases of PPCM revealed the presence of preeclampsia in $22 \%$ of patients [8].

However, there are controversies in the definition of PPCM, and some researchers state that heart failure in the course of any hypertensive disorder during pregnancy cannot be included in the definition of PPCM - they call it hypertensive heart failure of pregnancy (HHFP) [9].

On the other hand, it was recently suggested that PPCM may be associated with increased placental production of sFlt-1, as in preeclampsia. Serum sFlt-1 level is increased not only in women with preeclampsia, but also in patients suffering from PPCM without any hypertensive disorder (up to 10-fold higher than in the control group). In normal pregnancies sFlt-1 serum levels return to normal values within 48-72 hours after delivery. In patients developing PPCM postpartum sFlt-1 levels are still elevated four to six weeks after delivery, especially in women with preeclampsia [10].

\section{Future cardiovascular risk}

The syndrome of preeclampsia is now recognised as a risk factor for future cardiovascular disease. Epidemiological studies revealed that preeclampsia is associated with four-fold increased risk of heart failure, twofold increased risk of coronary artery disease, two-fold

Table 2. Future risk of cardiovascular, cerebrovascular, and kidney disease in women with preeclampsia [11, 12, 31-33]

\begin{tabular}{|c|c|c|}
\hline Event & $\begin{array}{c}\text { Study/ } \\
\text { meta-analysis }\end{array}$ & Risk \\
\hline Heart failure & Wu et al. & RR 4.19 (95\% Cl 2.09-8.38) \\
\hline $\begin{array}{l}\text { Coronary heart } \\
\text { disease }\end{array}$ & $\begin{array}{l}\text { Wu et al. } \\
\text { Berks et al. }\end{array}$ & $\begin{array}{l}\text { RR } 2.50(95 \% \text { Cl } 1.43-4.37) \\
\quad \text { RR } 1.89(\text { IQR } 1.76-1.98)\end{array}$ \\
\hline $\begin{array}{l}\text { Cardiovascular } \\
\text { disease death }\end{array}$ & Wu et al. & RR 2.21 (95\% Cl 1.83-2.66) \\
\hline Stroke & $\begin{array}{l}\text { Wu et al. } \\
\text { Berks et al. }\end{array}$ & $\begin{array}{l}\text { RR } 1.81(95 \% \text { Cl } 1.29-2.55) \\
\quad \text { RR } 1.55(\text { IQR } 1.40-1.71)\end{array}$ \\
\hline Microalbuminuria & McDonald et al. & RR 4.31 (95\% Cl 2.7-6.89) \\
\hline $\begin{array}{l}\text { End-stage renal } \\
\text { disease }\end{array}$ & Vikse et al. & $\begin{array}{c}\text { RR } 4.7(95 \% \mathrm{Cl} 3.6-6.1) \\
\text { PE in } 1^{\text {st }} \text { pregnancy } \\
\text { RR } 15.5(95 \% \mathrm{Cl} 7.8-30.8) \\
\text { PE during } 2^{\text {nd }} \text { or } 3^{\text {rd }} \\
\text { pregnancies } \\
\text { HR } 4.7(95 \% \mathrm{Cl} 3.6-6.0)\end{array}$ \\
\hline
\end{tabular}

increased risk of stroke, and two-fold increased risk of cardiovascular death later in life [11, 12] (Table 2).

It still remains unclear whether preeclampsia is an individual risk factor for future cardiovascular events or an early marker of women with high-risk profiles for cardiovascular disease. Risk factors for cardiovascular disorders and preeclampsia are very similar and include: obesity, dyslipidaemia, insulin resistance, pro-inflammatory and hypercoagulable state, and endothelial dysfunction. Thus, the pregnancy can only be a trigger for cardiovascular alterations that manifest in the development of preeclampsia.

On the other hand, there is strong evidence that changes in cardiovascular, endothelial, and metabolic systems occurring in the course of preeclampsia may not fully recover after delivery and can be a cause of future disease. Melchiorre et al. found that one year postpartum $70 \%$ of women with early-onset preeclampsia presented stage B (asymptomatic) heart failure and $40 \%$ developed hypertension within one or two years after delivery. It was quite different in the group of women with late-onset preeclampsia - only $24 \%$ presented stage B heart failure one year postpartum [7, 13].

In the study performed by Irgens et al. on a Norwegian cohort, with 13 years of follow-up, preeclampsia was associated with significantly higher risk of cardiovascular-related death. The highest association involved early-onset preeclampsia with preterm birth, whereas women with term preeclampsia had only a slight increase in cardiovascular-related death but no significant increase in stroke [14].

The study called CHAMPS, published in 2005, consisting of a cohort of 1 million women with maternal placental syndromes (preeclampsia, gestational hypertension, placental abruption, and FGR) followed for an average 8.7 years revealed additionally that women with pregnancies complicated by FGR or stillbirth had the highest risk of cardiovascular disease. It means that even typical obstetric complications in preeclampsia are related to elevated cardiovascular risk, and pregnancy can be found as a kind of "stress test" for the heart and the whole cardiovascular system [15].

For all these reasons, since 2011 the American Heart Association has included hypertensive disorders of pregnancy to major risks factors for cardiovascular diseases and recommends that obstetricians refer their patients to primary care physicians or cardiologists because they need adequate follow-up [16].

ESC in the 2016 European Guidelines on cardiovascular disease prevention in clinical practice suggests that in women with history of preeclampsia and/or gestational hypertension periodic screening for hypertension and diabetes mellitus should be performed [17].

Also, the ACOG recommends yearly assessment of BP, lipids, fasting blood glucose, and body mass index for women with medical history of preeclampsia who gave 
birth preterm (less than 37 weeks' gestation) or who have a medical history of recurrent preeclampsia [2].

\section{Cardiovascular risk in the offspring of preeclamptic women}

Concerning cardiovascular complications in preeclampsia, it should be mentioned that there is also evidence of increased cardiovascular risk in the offspring of preeclamptic mothers. Multiple studies have shown a correlation between maternal pregnancy hypertensive disorders and the development of hypertension in their children [11].

However, it is not clear if this relationship is caused by a direct effect, a familial aggregation of risk, or is confounded by its association with intrauterine growth restriction. A meta-analysis of case-control and cohort studies on the effect of maternal hypertension on offspring demonstrated an increase in systolic and diastolic blood pressure among children of preeclamptic women compared to normotensive women. The differences were observed even after adjusting for gestational age and birth weight, in both males and females. On the other hand, after adjustment for maternal body mass and blood pressure, the difference in SBP was largely attenuated $[18,19]$. It may suggest the influence of maternal, familiar, and genetic factors on blood pressure of the offspring and needs further research. Furthermore, there is also strong evidence for increased risk of developing preeclampsia in daughters and sisters of preeclamptic women [20].

Shared environmental and genetic factors of familiar aggregation pose a challenge to future studies examining cardiovascular risk in the offspring of women with preeclampsia.

\section{Future work}

It is important to notice that the risk of cardiovascular diseases is influenced by many factors appearing throughout life and there is a large time gap between pregnancy and development of cardiovascular disease in women. Therefore, there is a need for further research that can explain this connection. Most available studies in this area are based on national medical registries and thus have a retrospective nature; there are also differences in definitions of gestational hypertension and preeclampsia and also in follow-up time. That makes these studies open to bias. Only well-planned prospective research with standardised definitions including patients with similar risk factor profiles can add valuable weight to the body of evidence in this area.

\section{Cerebrovascular complications in preeclampsia}

Neurological complications with a close relationship to preeclampsia are: posterior reversible encephalopathy syndrome (PRES) and stroke.

\section{Nervous system in normal pregnancy and preeclampsia}

The nervous system undergoes numerous physiological adaptations during pregnancy. Most of all, they are a consequence of changes in other systems - markedly increased hormone levels, hypercoagulability, and cardiovascular modifications.

Substantial changes in maternal haemodynamics, together with alterations in coagulation profile, lead to cerebral endothelial permeability and pressure adaptations. The middle cerebral artery (MCA) perfusion pressure increases steadily during gestation and returns to nonpregnant values in the puerperium. Oestrogen lowers the seizure threshold by downregulating expression of GABA in the brain, whereas progesterone and its metabolites exert the opposite effect. Several animal studies indicate that the abrupt withdrawal of progesterone can be followed by increased seizure susceptibility. However, the relationship between progesterone and eclampsia has not been studied [21].

During normal pregnancy increased sympathetic nervous activity (SNA) can be observed. It is significantly higher during preeclampsia, and it is proposed that over-activation of the SNA contributes to altered uteroplacental vascular tone and perfusion leading to placental ischaemic events and exaggerated responses to soluble placenta ischaemic factors [21].

\section{Posterior reversible encephalopathy syndrome}

PRES was first described in 1996 by Hinchey et al. and is characterised by a range of clinical symptoms, such as headache, seizures, visual disturbances, and other focal neurological deficits accompanied by vasogenic cerebral oedema observed in magnetic resonance imaging (MRI) or computed tomography (CT), predominantly in the posterior regions [22].

There are two leading theories regarding the pathophysiology of PRES. First is elevation of blood pressure levels above the upper brain autoregulatory limit, leading to cerebral hypertension, vascular leakage, and vasogenic oedema. Normally, in healthy people, cerebral blood flow autoregulation is efficient between 50 and $150 \mathrm{~mm} \mathrm{Hg}$. Conditions like arterial hypertension or acute fluctuations of blood pressure may lead to increased vulnerability of the cerebral circulation and predispose to ischaemia. The second theory is endothelial dysfunction caused by circulating toxins: exogenic 
(chemotherapy agents, immunosuppression) and endogenic (preeclampsia, sepsis, autoimmune diseases). In this theory blood pressure elevations are a sequel of primary endothelial damage [22].

The exact prevalence of PRES in women with preeclampsia/eclampsia is difficult to estimate because of the small number of studies concerning it. In one retrospective study PRES was found in more than $90 \%$ of eclamptic and in about $20 \%$ of preeclamptic women with neurological symptoms [23].

It is important to note that there may be differences in patterns of PRES associated with preeclampsia and PRES associated with other conditions. Liman et al. showed in their retrospective study that patients with preeclampsia/ eclampsia had a significantly higher presence of headaches and significantly lower prevalence of altered mental status than nonpregnant women. However, there were no differences in the frequency of seizures or visual disturbances between the groups [22-24].

PRES is considered to be a reversible syndrome, because clinical signs and symptoms regress in the majority of patients. The prognosis is mainly determined by underlying pathology, and some neurological sequelae (e.g. epilepsy, cognitive difficulties related to memory and concentration) may persist. In long-term follow-up cerebral white matter lesions (WMLs) are diagnosed significantly more often in formerly preeclamptic/eclamptic women than in age-matched controls with uncomplicated pregnancy [24].

It seems that PRES in preeclampsia is associated with a less severe course of disease, but it still remains detrimental for young women demanding adequate diagnosis and treatment to prevent its long-term consequences [22].

\section{Stroke}

Stroke is defined as a local or global alteration in cerebral function lasting over 24 hours and is a devastating diagnosis because it can result in long-term disability and death.

The prevalence of stroke during pregnancy and postpartum is estimated at 34 per 100,000 deliveries and is still increasing. In the United States the rate of pregnancy-associated stroke rose from $45 \%$ (in the years $1994-$ 1995 ) to $83 \%$ (in the years $2005-2006$ ). The mortality rate is 1.4 per 100,000 deliveries [25].

The frequencies of ischaemic and haemorrhagic stroke during pregnancy have been reported as similar. However, there are also studies that have concluded that ischaemic stroke due to cerebral sinus thrombosis is the most common type of stroke during pregnancy and puerperium [26].

The most important risk factors for pregnancy-associated stroke are: hypertensive disorders during pregnancy, chronic hypertension, pregestational diabetes, caesarean delivery, heart disease, thrombophilia, and migraine headaches. Too et al. estimated that the risk of postpartum stroke within 60 days after delivery was 41.7 per 100,000 deliveries for patients with hypertensive disorders of pregnancy, 59.6 per 100,000 deliveries for patients with chronic hypertension without superimposed preeclampsia, and 21.7 per 100,000 deliveries for patients without either condition [25].

In the course of severe preeclampsia/eclampsia the most common type of stroke is haemorrhagic stroke as a result of severe hypertension. In the study conducted by Martin et al. (only with women affected by preeclampsia/eclampsia) the prevalence of haemorrhagic stroke was $89 \%$ [27]. However, there are available studies reporting an increased risk of ischaemic stroke in preeclampsia as well [25].

The clinical presentation in stroke associated with preeclampsia/eclampsia is quite different than in stroke due to other reasons. The most common symptoms of stroke in this group of pregnant women are: severe headache, impairment of consciousness and elevated systolic blood pressure. Some studies have confirmed that an acute increase in systolic blood pressure (SBP) is the most important risk factor for haemorrhagic stroke in preeclampsia. Despite the fact that the majority of international guidelines emphasise DBP as a primary determinant of intervention, women with severe preeclampsia and high isolated SBP should be immediately treated with antihypertensive therapy because of a very high risk for haemorrhagic stroke [25].

Recent studies suggest an $80 \%$ increased risk of stroke in patients with preeclampsia compared to women with uncomplicated pregnancy. What is very important - preeclampsia increases also (by about 55-60\%) the risk of future non-pregnancy-related stroke [22] (Table 2).

\section{Future work}

Several factors including modern lifestyle, environment, and postponing the age of childbearing lead to increasing incidence of neurological pregnancy complications, such as stroke. There is a need for further research in this area, which aims to work out effective and detailed preventive strategies for these patients.

\section{Renal dysfunction and preeclampsia}

The most common renal complications related to preeclampsia include: renal failure, acute kidney injury, and future risk of end-stage renal disease.

\section{Renal function during uncomplicated pregnancy}

Renal function undergoes a lot of physiological changes during pregnancy. The overall size of the human kidney 
increases due to a rise in renal vascular and interstitial volume. In more than $80 \%$ of normal pregnancies a dilation of the renal pelvises can be observed, due to external compression of the ureter, as well as hormonal changes related to progesterone [28].

Renal perfusion increases during normal pregnancy because of increased cardiac output, and it leads to a rise in glomerular filtration rate (GFR) - about 40-60\% in the second half of pregnancy. Serum creatinine falls during normal pregnancy to a normal range of 0.4-0.8 $\mathrm{mg} / \mathrm{dl}$. A serum creatinine of $1 \mathrm{mg} / \mathrm{dl}$ (considered as normal in nonpregnant women) can represent a renal impairment in pregnant women. Protein excretion during normal pregnancy increases, but does not exceed $300 \mathrm{mg}$ per day [28].

\section{Renal function during preeclampsia}

During preeclampsia functional changes in renal haemodynamics are quite different. The GFR in women with preeclampsia is significantly lower than in healthy controls. It is related to typical histopathological changes including fibrin deposition, endothelial swelling, and loss of capillary space. These changes, called "glomerular endotheliosis" (a term introduced by Spargo in 1976), are pathognomonic for preeclampsia, which is considered as the most common glomerular disease worldwide. Nevertheless, in the majority of patients with preeclampsia glomerular changes disappear within eight weeks of delivery [28].

During normal pregnancy changes in renin-angiotensin-aldosterone system (RAAS) are also observed, including elevated angiotensin II (ang-II) levels and decreased sensitivity to it. In contrast, during preeclampsia decreased components of the RAAS and decreased sensitivity to ang-II have been reported. It is probably related to increased placental ang-II type 1 receptor $\left(A T_{1} R\right)$ expression and circulating $A T_{1} R$ autoantibodies. Moreover, circulating AT $R$ antibodies and ang-II are capable of inducing sFlt-1 production by $\mathrm{AT}_{1} \mathrm{R}$ activation. It means they can be responsible for placental sFlt-1 up-regulation in preeclampsia [29].

\section{Acute kidney injury in preeclampsia}

In rare cases, preeclampsia can lead also to renal cortical necrosis or acute tubular necrosis and is one of the main causes of acute renal failure in pregnancy.

Acute kidney injury (AKI) is defined as an increase in serum creatinine level in patients without chronic kidney disease and manifests most of all as oliguria or anuria. In a Chinese cohort study the incidence of AKI during pregnancy and puerperium was $0.81 \%$. Preeclampsia and postpartum haemorrhage were the main cause of it. About $17 \%$ of patients with preeclampsia/eclampsia and $60 \%$ of women with HELLP syndrome were diagnosed with AKI. The mortality rate among pregnant women with AKI was $4.08 \%$, but the major causes of death were amniotic fluid embolism and postpartum haemorrhage, whereas the maternal outcome of AKI related to preeclampsia was relatively good [30].

\section{Future risk of end-stage renal disease}

As mentioned before, after preeclampsia, complete renal recovery takes place in the majority of cases. However, there is strong evidence for increased risk of microalbuminuria and end-stage renal disease in future life after pregnancy complicated by preeclampsia (Table 2 ).

A recent meta-analysis revealed that $31 \%$ of preeclamptic women had microalbuminuria after seven years postpartum compared to $7 \%$ of women with uncomplicated pregnancy. The risk of developing microalbuminuria was increased four-fold after mild preeclampsia and eight-fold after severe preeclampsia [31].

End-stage renal disease (ESRD) is chronic renal failure requiring long-term dialysis or renal transplantation. In a cohort of 570,433 women, in a study performed by Vikse et al., preeclampsia in the first pregnancy was associated with a relative risk (RR) of ESRD of 4.7. If women also had preeclampsia during subsequent pregnancies the RR of ESRD was 15.5 [32].

In the Chinese cohort of 13,633 women with hypertensive disorders of pregnancy (HDPs) in the follow-up period of nine years on average, $0.34 \%$ of women developed ESRD compared to $0.02 \%$ of women with uncomplicated pregnancy. The risk of ESRD in women with HDPs was 4-15-times higher than in healthy controls, and it was even higher in women who had a preterm birth or low birth weight child. The risk was highest in women with history of preeclampsia superimposed on chronic hypertension (hazard ratio 44.72) [33].

\section{Future work}

Several factors may explain the increased risk of microalbuminuria and ESRD in women with preeclampsia: shared risk factors of preeclampsia and kidney disease, undetected hypertension or microalbuminuria before pregnancy, and the impact of preeclampsia itself on kidney function with incomplete recovery after glomerular endotheliosis. Similarly as for future cardiovascular risk, there is a need for well-designed prospective studies that might help to explain this connection.

\section{Liver failure and preeclampsia}

The most important liver abnormalities with a close relationship to preeclampsia are liver failure and hepatic rupture. 


\section{Liver function during normal pregnancy}

During normal pregnancy many physiological and hormonal changes occur, and some of them are also related to liver function.

Despite the rise in cardiac output, blood flow to the liver remains constant. The liver in not enlarged and impalpable. Due to compression of the inferior vena cava by the enlarging uterus small, clinically insignificant oesophageal varices can occur in up to $50 \%$ of pregnancies during the late second and third trimester [34].

Biochemical liver function tests are also altered during pregnancy. A mild increase in alkaline phosphatase (due to placental isoenzyme) and a mild decrease in serum albumin levels (due to increased plasma volume and haemodilution) can be observed. Urea, haemoglobin levels, and prothrombin time remain unchanged or slightly decreased due to haemodilution. Elevations in transaminases, bilirubin, or prothrombin time are abnormal and require further diagnostics. Liver function test abnormalities occur in about $3 \%$ of pregnancies [34].

\section{Liver dysfunction during preeclampsia}

Preeclampsia/eclampsia and HELLP syndrome are the main cause of hepatic dysfunction during pregnancy. Abnormal biochemical liver tests occur in about 20$30 \%$ of these patients [34].

In the study performed by Suresh et al. the overall incidence of hepatic dysfunction during pregnancy was $3.2 \%$ and the most common causes of it were: preeclampsia (1.8\%), eclampsia (0.6\%), HELLP syndrome $(0.24 \%)$, viral infection $(0.19 \%)$, hyperemesis gravidarum $(0.14 \%)$, ICP (0.13\%), and chronic liver disease $(0.03 \%)$. Liver dysfunction was also related to premature deliveries, low birth weight babies, and increased maternal and foetal mortality [35].

Liver dysfunction during preeclampsia is related (as in involvement of other organs) to endothelial dysfunction, which leads to hepatic microcirculatory deterioration and hepatocellular necrosis. Liver biopsies in women with HELLP syndrome have shown thrombotic microangiopathy [36].

There is also evidence of the contribution of sFlt-1 in the development of liver dysfunction in preeclampsia. SFlt-1 antagonises VEGF, whereas VEGF increases the expression of endothelial nitric oxide synthase (eNOS) and activates it. Raised sFlt-1 production during preeclampsia and therefore the lack of eNOS synergistically induce liver dysfunction and thrombocytopaenia, which was confirmed in animal models. The exact mechanism of liver injury is unknown, but some studies demonstrated also that placenta-derived CD95 ligand (Fas) causes damage in hepatocytes and leads to their apoptosis in women with HELLP syndrome [37].

\section{Hepatic rupture}

One of the most important hepatic complications related to hypertensive disorders of pregnancy is hepatic haemorrhage and liver rupture.

It is a very rare but life-threatening complication with very high maternal and perinatal mortality rates (39\% and $42 \%$, respectively). The incidence of liver rupture in pregnancy is estimated on 1 per 67,000 deliveries and 1 per 2000 patients with preeclampsia or HELLP syndrome. Additional risk factors in this group of patients are: multiparity, age $>40$ years, and gestational age $>32$ weeks. The pathophysiology has been attributed to vasoconstriction due to increased levels and sensitivity to circulating vasopressors, such as endothelin and angiotensin II, leading to ischaemia, necrosis, and finally rupture [38].

Liver rupture during pregnancy may occur not only because of hypertensive disorders, but also due to coexisting hepatic pathologies - adenomas, haemangiomas, malignancies, and coagulation disorders. However, they are rather casuistic [38].

Escobar Vidarte et al. in their literature review reported 35 cases of pregnancy hepatic haemorrhage: 28 (80\%) of them caused by HELLP syndrome, three $(8.5 \%)$ caused by preeclampsia and HELLP syndrome, and four (11.4\%) by preeclampsia without HELLP syndrome [38].

Clinical presentation of hepatic haemorrhage includes epigastric right upper-quadrant pain, hypovolemic shock, and cardiovascular collapse. In the vast majority of patients (approximately 90\%) abdominal pain is the first symptom, and it should always raise suspicion. Computed tomography or magnetic resonance imaging is the investigation of choice. Patients with liver rupture require urgent laparotomy, because only surgery significantly reduces maternal mortality [39].

\section{Future work}

Hepatic rupture is rare, but it is connected to high mortality rate, and there is still a lack of detailed prevention strategies to avoid this complication. There is also a need for further research estimating potential relationship between preeclampsia and liver abnormalities in future life, years after delivery.

\section{Coagulopathy and preeclampsia}

Nankali et al. in their cohort of 349 severe preeclampsia cases presented coagulopathy as the most frequent maternal complication (10.6\% of patients). The most common coagulopathies in preeclampsia are thrombocytopaenia and DIC [40]. 


\section{Coagulation-fibrinolytic system during normal pregnancy and preeclampsia}

Pregnancy is recognised as a procoagulant state because of an increase in concentration of fibrinogen and other clotting factors together with a reduction of levels of natural anticoagulants, such as protein S and C [41].

During preeclampsia the coagulation-fibrinolytic system is seriously affected by maternal inflammatory reactions and immune dysfunction. Increase in blood clotting is crucial for pregnant women to reduce postpartum haemorrhage. When the balance between coagulation and fibrinolysis is altered, as in preeclampsia, the blood flow of the placenta and other organs is blocked by microthrombosis. In comparison to normal pregnancy, significantly higher levels of tissue factor, fibrinogen, and von Willebrand factor and significantly lower levels of anticoagulation factors, such as antithrombin and tissue factor pathway inhibitor 2 (TFPI2), are observed in women with preeclampsia. Thus, preeclampsia is sometimes called a "super-hypercoagulable state" [42].

\section{D-dimer in pregnancy}

D-dimer is a specific degradative product coming from hydrolysis of the fibrin monomer and thus serves as an indirect marker for thrombosis and fibrinolytic activity, widely used in the diagnosis of venous thromboembolism (VTE).

In normal pregnancy, however, the maternal D-dimer concentration increases progressively from conception to delivery, and for this reason the D-dimer test has no use in ruling out VTE with standard cut-off of $0.5 \mathrm{mg} / \mathrm{l}$. Kline et al. established a mean D-dimer concentration in normal pregnancy of $0.579 \mathrm{mg} / \mathrm{l}$ in the first trimester, $0.832 \mathrm{mg} / \mathrm{l}$ in the second trimester, and $1.159 \mathrm{mg} / \mathrm{l}$ in the third trimester. The mean rise in the maternal D-dimer concentration from preconception to the third trimester was $0.69 \mathrm{mg} / \mathrm{l}$ [43].

In the recent study (published in 2018) plasma D-Dimer levels were measured during the three trimesters of pregnancy to establish trimester-specific reference ranges. D-Dimer levels increased progressively during pregnancy with the highest values in the third trimester, when in $99 \%$ of women included in the study they were above the conventional cut-off point $(500 \mu \mathrm{g} / \mathrm{l})$ [44].

It is also important to note that in women with preeclampsia D-dimer concentrations are additionally significantly higher than in healthy pregnant controls, especially in severe early-onset preeclampsia. In women with preeclampsia D-dimer is considered to involve in the dynamic balance between plasminogen activators (t-PA and UPA) and plasminogen inhibitor (PAI-1). However, according to new guidelines, D-dimer plasma concentration should not be routinely measured during pregnancy because of the lack of reliable norms and possible confusion in diagnostics [45].

\section{Thrombocytopaenia during preeclampsia}

Thrombocytopaenia is defined as a platelet count below $150,000 / \mu \mathrm{l}$ and is the second most common abnormality of complete blood count in pregnancy (beside anaemia) with a prevalence of 6.6 to $11.6 \%$ in the third trimester [41].

Preeclampsia and HELLP syndrome are responsible for $15-22 \%$ of all cases of thrombocytopaenia in pregnancy. On the other hand, thrombocytopaenia is diagnosed in $30-50 \%$ of women with preeclampsia and is even one of the diagnostic criteria of severe preeclampsia and HELLP syndrome [46].

There is a significant association between severity of thrombocytopaenia and maternal morbidity. If the platelet count is in the range of $100-150,000 / \mu \mathrm{l}$, the rate of maternal complications is $40 \%$. For the range of $50-100,000 / \mu \mathrm{l}$ it is $54 \%$, and for platelet count below $50,000 / \mu$ it is $64 \%$. Fortunately, fewer than $5 \%$ of preeclamptic women develop severe thrombocytopaenia with platelet count below 50,000/ $\mu$ l [46].

Thrombocytopaenia that develops during preeclampsia or HELLP syndrome is related to excessive platelet activation due to endothelial dysfunction and proinflammatory cytokines, does not cause neonatal thrombocytopaenia, and usually normalises in 6-11 days postpartum [46].

\section{Disseminated intravascular coagulation syndrome and preeclampsia}

DIC is a thrombo-haemorrhagic condition secondary to underlying clinical pathologies characterised by activation of intravascular coagulation accompanied by secondary fibrinolysis.

Several pregnancy complications may lead to DIC, including: preeclampsia and HELLP syndrome, placental abruption, intrauterine foetal death, amniotic fluid embolism, placenta accreta, and acute fatty liver of pregnancy [47].

Preeclampsia and HELLP syndrome are leading causes of DIC in developing countries, whereas in developed countries they are placental abruption and postpartum haemorrhage. DIC is reported in 12-14\% of preeclamptic women. However, it has been suggested that in the majority of cases these women also had HELLP syndrome, and the prevalence of DIC in women with preeclampsia only is rare [42].

There are several mechanisms of developing DIC in patients with preeclampsia and HELLP syndrome: consumption coagulopathy (because of strong association of HELLP and placental abruption), hepatic injury (de- 
creased production of clotting factors), and systemic maternal inflammatory response (characteristic for preeclampsia) [42].

Significantly increased circulating pro-inflammatory cytokines during preeclampsia can lead to exaggerated expression of tissue factor by leukocytes and endothelial cells. There is strong evidence that tissue factor plasma levels are markedly higher in women with preeclampsia compared with healthy controls [42].

There is also a suggestion that in women who develop DIC associated with HELLP syndrome there is an inherit imbalance between coagulation factors, anti-coagulation proteins, and reduced fibrinolysis. This state was defined by de Boer et al. as "compensated DIC", which can easily developed into real DIC in the presence of other risk factors. Women with HELLP syndrome should be closely followed for the development of DIC, because this coagulopathy is associated with the highest risk of maternal and foetal mortality [48].

\section{Future work}

Coagulopathies during preeclampsia are well-described but remain a significant cause of maternal morbidity and mortality. Therefore, researchers and clinicians should maximise their efforts for early diagnosis and appropriate therapy in these group of preeclampsia complications.

\section{Conclusions}

Preeclampsia is a multisystem disorder of pregnancy that remains a leading cause of maternal and foetal mortality. It is still an underestimated risk factor for future cardiovascular, cerebrovascular, and kidney diseases. There is a need for early screening of preeclampsia and effective prophylaxis to prevent the most severe complications related to increased risk of maternal death and disability. On the other hand, women with pregnancy complicated by preeclampsia should also be covered by a very watchful follow-up, many years after pregnancy, because of rising cardiovascular disease-related mortality in adult women, especially those aged 35-45 years.

\section{Disclosure}

The authors report no conflict of interest.

\section{References}

1. Regitz-Zagrosek V, Roos-Hesselink JW, Bauersachs J, et al. 2018 ESC Guidelines for the management of cardiovascular diseases during pregnancy. Eur Heart J 2018; 39: 3165-3241.

2. Hypertension in pregnancy. American College of Obstetricians and Gynecologists; 2013.
3. Chaiworapongsa T, Chaemsaithong P, Yeo L, Romero R. Pre-eclampsia part 1: current understanding of its pathophysiology. Nat Rev Nephrol 2014; 10: 466-480.

4. Henderson JT, Whitlock EP, O'Connor E, et al. Low-dose aspirin for prevention of morbidity and mortality from preeclampsia: a systematic evidence review for the U.S. Preventive Services Task Force. Ann Intern Med 2014; 160: 695-703.

5. Vaught AJ, Kovell LC, Szymanski LM, et al. Acute Cardiac Effects of Severe Pre-Eclampsia. J Am Coll Cardiol. 2018; 72: 1-11.

6. Orabona R, Sciatti E, Prefumo F, et al. Pre-eclampsia and heart failure: a close relationship. Ultrasound Obstet Gynecol Off J Int Soc Ultrasound Obstet Gynecol 2018; 52: 297-301.

7. Melchiorre K, Sharma R, Thilaganathan B. Cardiovascular implications in preeclampsia: an overview. Circulation. 2014; 130: 703-714.

8. Arany Z, Elkayam U. Peripartum Cardiomyopathy. Circulation 2016; 133 : 1397-1409.

9. Ntusi NBA, Badri M, Gumedze F, et al. Pregnancy-Associated Heart Failure: A Comparison of Clinical Presentation and Outcome between Hypertensive Heart Failure of Pregnancy and Idiopathic Peripartum Cardiomyopathy. PloS One 2015; 10: e0133466.

10. Damp JA, Arany Z, Fett JD, et al. Imbalanced Angiogenesis in Peripartum Cardiomyopathy (PPCM). Circ J Off J Jpn Circ Soc 2018; 82: 2689.

11. Wu P, Haththotuwa R, Kwok CS, et al. Preeclampsia and Future Cardiovascular Health: A Systematic Review and Meta-Analysis. Circ Cardiovasc Qual Outcomes 2017; 10: pii: e003497.

12. Berks D, Hoedjes M, Raat $H$, et al. Risk of cardiovascular disease after pre-eclampsia and the effect of lifestyle interventions: a literaturebased study. BJOG Int J Obstet Gynaecol 2013; 120: 924-931.

13. Ghossein-Doha C, van Neer J, Wissink B, et al. Pre-eclampsia: an important risk factor for asymptomatic heart failure. Ultrasound Obstet Gynecol Off J Int Soc Ultrasound Obstet Gynecol 2017; 49: 143-149.

14. Irgens HU, Reisaeter L, Irgens LM, Lie RT. Long term mortality of mothers and fathers after pre-eclampsia: population based cohort study. BMJ 2001; 323: 1213-1217.

15. Ray JG, Vermeulen MJ, Schull MJ, Redelmeier DA. Cardiovascular health after maternal placental syndromes (CHAMPS): population-based retrospective cohort study. Lancet Lond Engl 2005; 366: 1797-1803.

16. Whelton PK, Carey RM, Aronow WS, et al. 2017 ACC/AHA/AAPA/ABC/ ACPM/AGS/APhA/ASH/ASPC/NMA/PCNA Guideline for the Prevention, Detection, Evaluation, and Management of High Blood Pressure in Adults: Executive Summary: A Report of the American College of Cardiology/American Heart Association Task Force on Clinical Practice Guidelines. Hypertension 2018; 71: 1269-1324.

17. Piepoli MF, Hoes AW, Agewall S, et al. 2016 European Guidelines on cardiovascular disease prevention in clinical practice: The Sixth Joint Task Force of the European Society of Cardiology and Other Societies on Cardiovascular Disease Prevention in Clinical Practice (constituted by representatives of 10 societies and by invited experts)Developed with the special contribution of the European Association for Cardiovascular Prevention \& Rehabilitation (EACPR). Eur Heart J 2016; 37: 2315-2381.

18. Øglaend B, Forman MR, Romundstad PR, et al. Blood pressure in early adolescence in the offspring of preeclamptic and normotensive pregnancies. J Hypertens 2009; 27: 2051-2054.

19. Ferreira I, Peeters LL, Stehouwer CDA. Preeclampsia and increased blood pressure in the offspring: meta-analysis and critical review of the evidence. J Hypertens 2009; 27: 1955-1959.

20. Bartsch E, Medcalf KE, Park AL, et al. Clinical risk factors for pre-eclampsia determined in early pregnancy: systematic review and meta-analysis of large cohort studies. BMJ 2016; 353: i1753.

21. Lee LK. Physiological adaptations of pregnancy affecting the nervous system. Semin Neurol 2007; 27: 405-410.

22. McDermott M, Miller EC, Rundek T, et al. Preeclampsia: Association With Posterior Reversible Encephalopathy Syndrome and Stroke. Stroke 2018; 49: 524-530.

23. Liman TG, Bohner G, Heuschmann PU, et al. The clinical and radiological spectrum of posterior reversible encephalopathy syndrome: the retrospective Berlin PRES study. J Neurol 2012; 259: 155-164.

24. Postma IR, Slager S, Kremer HPH, et al. Long-term consequences of the posterior reversible encephalopathy syndrome in eclampsia and preeclampsia: a review of the obstetric and nonobstetric literature. Obstet Gynecol Surv. 2014; 69: 287-300. 
25. Too G, Wen T, Boehme AK, et al. Timing and Risk Factors of Postpartum Stroke. Obstet Gynecol 2018; 131: 70-78.

26. Crovetto F, Somigliana E, Peguero A, Figueras F. Stroke during pregnancy and pre-eclampsia. Curr Opin Obstet Gynecol 2013; 25: 425-432.

27. Martin JN, Thigpen BD, Moore RC, et al. Stroke and severe preeclampsia and eclampsia: a paradigm shift focusing on systolic blood pressure. Obstet Gynecol 2005; 105: 246-254.

28. Gonzalez Suarez ML, Kattah A, Grande JP, Garovic V. Renal Disorders in Pregnancy: Core Curriculum 2019. Am J Kidney Dis 2019; 73: 119-130;

29. van der Graaf AM, Toering TJ, Faas MM, Lely AT. From preeclampsia to renal disease: a role of angiogenic factors and the renin-angiotensin aldosterone system? Nephrol Dial Transplant 2012; 27 Suppl 3: iii51-57.

30. Mahesh E, Puri S, Varma V, et al. Pregnancy-related acute kidney injury: An analysis of 165 cases. Indian J Nephrol 2017; 27: 113-117.

31. McDonald SD, Han Z, Walsh MW, et al. Kidney disease after preeclampsia: a systematic review and meta-analysis. Am J Kidney Dis 2010; 55: 1026-1039.

32. Vikse BE, Irgens LM, Leivestad T, et al. Preeclampsia and the risk of endstage renal disease. N Engl J Med 2008; 359: 800-809.

33. Dai L, Chen Y, Sun W, Liu S. Association Between Hypertensive Disorders During Pregnancy and the Subsequent Risk of End-Stage Renal Disease: A Population-Based Follow-Up Study. J Obstet Gynaecol Can 2018; 40: 1129-1138.

34. Westbrook RH, Dusheiko G, Williamson C. Pregnancy and liver disease. J Hepatol 2016; 64: 933-945.

35. Suresh I, Tr V, Hp N. Predictors of Fetal and Maternal Outcome in the Crucible of Hepatic Dysfunction During Pregnancy. Gastroenterol Res 2017; 10: 21-27.

36. Perronne L, Dohan A, Bazeries P, et al. Hepatic involvement in HELLP syndrome: an update with emphasis on imaging features. Abdom Imaging 2015; 40: 2839-2849.

37. Oe Y, Ko M, Fushima T, et al. Hepatic dysfunction and thrombocytopenia induced by excess sFlt1 in mice lacking endothelial nitric oxide synthase. Sci Rep 2018; 8: 102.

38. Escobar Vidarte MF, Montes D, Pérez A, et al. Hepatic rupture associated with preeclampsia, report of three cases and literature review. J Matern Fetal Neonatal Med 2019; 32: 2767-2773.

39. Guo Q, Yang Z, Guo J, et al. Hepatic infarction induced by HELLP syndrome: a case report and review of the literature. BMC Pregnancy Childbirth 2018; 18: 191.

40. Nankali A, Malek-Khosravi S, Zangeneh M, et al. Maternal complications associated with severe preeclampsia. J Obstet Gynaecol India 2013; 63: 112-115.

41. Han L, Liu X, Li H, et al. Blood coagulation parameters and platelet indices: changes in normal and preeclamptic pregnancies and predictive values for preeclampsia. PloS One 2014; 9: e114488.

42. Erez O, Mastrolia SA, Thachil J. Disseminated intravascular coagulation in pregnancy: insights in pathophysiology, diagnosis and management. Am J Obstet Gynecol 2015; 213: 452-463.

43. Kline JA, Williams GW, Hernandez-Nino J. D-dimer concentrations in normal pregnancy: new diagnostic thresholds are needed. Clin Chem 2005; 51: 825-829.

44. Gutiérrez García I, Pérez Cañadas P, Martínez Uriarte J, et al. D-dimer during pregnancy: establishing trimester-specific reference intervals. Scand I Clin Lab Invest 2018; 78: 439-442.

45. Bozkurt M, Yumru AE, Sahin L, Salman S. Troponin I and D-Dimer levels in preeclampsia and eclampsia: prospective study. Clin Exp Obstet Gynecol 2015; 42: 26-31.

46. Jodkowska A, Martynowicz H, Kaczmarek-Wdowiak B, Mazur G. Thrombocytopenia in pregnancy - pathogenesis and diagnostic approach. Postepy Hig Med Dosw 2015; 69: 1215-1221.

47. Cunningham FG, Nelson DB. Disseminated Intravascular Coagulation Syndromes in Obstetrics. Obstet Gynecol 2015; 126: 999-1011.

48. de Boer K, ten Cate JW, Sturk A, et al. Enhanced thrombin generation in normal and hypertensive pregnancy. Am J Obstet Gynecol 1989; 160: 95-100. 